\title{
Effect of Two Temperatures on Reflection Coefficient in Micropolar Thermoelastic with and without Energy Dissipation Media
}

\author{
Rajneesh Kumar, ${ }^{1}$ K. D. Sharma, ${ }^{2}$ and S. K. Garg ${ }^{3}$ \\ ${ }^{1}$ Department of Mathematics, Kurukshetra University, Kurukshetra, India \\ ${ }^{2}$ Department of Mathematics, Swami Devi Dyal Institute of Engineering \& Technology, Barwala, India \\ ${ }^{3}$ Department of Mathematics, Deenbandhu Chhotu Ram University, Murthal, Sonipat, India \\ Correspondence should be addressed to K. D. Sharma; kd_sharma33@rediffmail.com
}

Received 9 April 2013; Accepted 29 September 2013; Published 16 February 2014

Academic Editor: Abdelkrim Khelif

Copyright (C) 2014 Rajneesh Kumar et al. This is an open access article distributed under the Creative Commons Attribution License, which permits unrestricted use, distribution, and reproduction in any medium, provided the original work is properly cited.

The reflection of plane waves at the free surface of thermally conducting micropolar elastic medium with two temperatures is studied. The theory of thermoelasticity with and without energy dissipation is used to investigate the problem. The expressions for amplitudes ratios of reflected waves at different angles of incident wave are obtained. Dissipation of energy and two-temperature effects on these amplitude ratios with angle of incidence are depicted graphically. Some special and particular cases are also deduced.

\section{Introduction}

The theory of micropolar elasticity was introduced and developed by Eringen [1]. The theory of micropolar continuum mechanics gives consideration to the microstructure. Micropolar theory is useful in structure materials with a fibrous, lattice, or granular micropolar structure. The main difference of micropolar elastic material from the classical elastic material is that each point has extra rotational degrees of freedom independent of translation and the material can transmit couple stress as well as usual force stress.

The linear theory of micropolar thermoelasticity has been developed by extending the theory of micropolar continua to include thermal effect and comprehensive review work on the subject was given by Eringen [2,3] and Nowacki [4]. Dost and Taborrok [5] presented the generalized thermoelasticity by using Green and Lindsay theory. Chandrasekharaiah [6] developed a heat flux dependent micropolar thermoelasticity. Boschi and Iesan [7] presented a generalized theory of micropolar thermoelasticity that permits the transmission of heat as thermal waves at finite speed.

The main difference of thermoelasticity with two temperatures with respect to the classical one is the thermal dependence. Chen et al. [8, 9] have formulated a theory of heat conduction in deformable bodies, which depends on two distinct temperatures, the conductive temperature $\Phi$ and thermodynamic temperature $T$. For time independent situations, the difference between these two temperatures is proportional to the heat supply. For time dependent problems in wave propagation the two temperatures are in general different. The two temperatures $T, \Phi$ and the strain are found to have representation in the form of a travelling wave pulse, a response which occurs instantaneously throughout the body (Boley and Tolins [10]). The wave propagation in the twotemperature theory of thermoelasticity was investigated by Warren and Chen [11].

Youssef [12] constructed the new theory of generalized thermoelasticity by taking into account the theory of heat conduction in deformable bodies, which depends on two distinct temperatures, the conductive temperature and the thermodynamic temperature, where the difference between these two temperatures is proportional to the heat supply and proves the uniqueness theorem. Youssef and Bassiouny [13] solved the boundary value problem of one dimension in two-temperature generalized thermopiezoelastic half space, subjected to the thermal shock by using state space approach. 
Bassiouny and Youssef [14] investigated the boundary value problem of one-dimensional finite piezoelastic road with loading on its boundary with different types of loading in two-temperature generalized thermoelasticity. Ezzat and Bary [15] adopted the state space approach to obtain the solution of one-dimensional problems for any set of boundary conditions in magnetothermoelastic medium with two temperatures.Mukhopadhyay and Kumar (2009) investigated the thermoelastic interactions in an infinite medium with a cylindrical cavity in the context of a theory of generalized thermoelasticity in which theory of heat conduction in deformable bodies depends on two different temperaturesconductive temperature and dynamic temperature. Kumar et al. [16] established the variational and reciprocal principles in the context of linear theory of two-temperature generalized thermoelasticity, for a homogeneous and isotropic body. Ezzat and Awad [17] proved uniqueness theorem and thermal shock problem in micropolar thermoelastic medium with two temperatures. Kumar and Mukhopadhyay [18] investigated the plane wave propagation under two-temperature thermoelastic medium. Kaushal et al. [19] studied propagation of waves in generalized thermoelastic continua with two temperatures.

Kaushal et al. [20] discussed wave propagation in temperature rate dependent thermoelasticity with two temperatures. Shaw and Mukhopadhyay [21] derived the variational principle and reciprocity theorem in generalized micropolar thermoelasticity with two temperatures by using fractional calculus. Youssef [22] proved the uniqueness theorem in the theory of Thermoelastic with two temperatures and without energy dissipation. El-Karamany [23] proved the reciprocal theorem and convolutional variational principle in a inhomogeneous micropolar thermoviscoelastic solid with two temperatures. El-Karamany and Ezzat [24] proved the uniqueness and reciprocal theorems in a linear anisotropic and inhomogeneous thermoelastic centrosymmetric solid in the two-temperature Green-Naghdi theories of type three. Mukhopadhyay et al. [25] proved the uniqueness and reciprocal theorem in the theory of two-temperature thermoelasticity with two-phase lags. Ezzat et al. [26] constructed the new mathematical model of two-temperature magnetothermoelasticity in which the fractional order dual-phase-lag heat conduction law is considered. Singh and Bala [27] studied the propagation of plane waves in an isotropic generalized thermoelastic solid half space with two temperatures. Ezzat et al. [26] introduced both modified Ohm's and Fourier's laws to solve the equations of the linear theory of magneto-thermoviscoelasticity involving two-temperature theory, allowing the second sound effects. Youssef [28] derived the variational principal theorem for a homogeneous isotropic body in the theory of two temperatures without energy dissipation. Banik and Kanoria [29] investigated thermoelastic interaction in a piezoelastic half-space body in which the boundary is stressfree and subjected to thermal loading in the context of twotemperature generalized thermoelasticity.

Ezzat and Othman [30] studied the plane wave propagation in Electromagneto thermoelastic with two relaxation time in a medium of perfect conductivity by using normal mode analysis. Ezzat et al. [31] investigated the plane wave propagation in Electromagneto thermoelastic with thermal relaxation time by using normal mode analysis. Othman [32] investigated the effect of rotation on plane wave propagation in the context of generalized theory of thermoelasticity with two relaxation times and obtained the expressions for the temperature distribution, the displacement components, and thermal stress by using normal mode analysis. Two-dimensional coupled problem in electromagnetothermoelasticity for thermally and electrically conducting half space solid by using normal mode analysis was investigated by $\mathrm{He}$ and $\mathrm{Li}$ [33]. Othman et al. [34] studied the effect of rotation on plane wave propagation in the context of Green's Naghdi theory type II by using the normal mode analysis. Ezzat and Awad [35] adopted the normal mode analysis technique to obtain the temperature gradient, displacement, stress, couple stresses, microrotation, electric field, magnetic field, and current density in micropolar generalized magnetothermoelasticity by using modified Ohm's and Fourier's law. Othman et al. [36] studied the effect of diffusion on two-dimensional problem of generalized thermoelasticity with Green-Naghdi theory and obtained the expressions for displacement components, stresses, temperature fields, concentration, and chemical potential by using normal mode analysis. Othman et al. [37] used normal mode technique to obtain the expressions for displacement components, force stresses, temperature, couple stresses, and microstress distribution in a thermomicrostretch elastic medium with temperature dependent properties for different theories. Othman and Lotfy [38] studied the plane wave propagation in microstretch thermoelastic half space by using normal mode analysis. Kumar et al. [39] investigated the disturbance due to force in normal and tangential direction by using normal mode analysis in fluid saturated porous medium. Kumar et al. [40] investigated the effect of viscosity on plane wave propagation in heat conducting transversely isotropic micropolar viscoelastic half space.

To study the propagation of thermal waves at finite speed, it may be possible in the foreseeable future to identify an idealized material. Green and Naghdi [41-43] have made relevant theoretical development in the theory of thermoelasticity and provided sufficient basic modifications in the constitutive equations that allow treatment of wider class of heat flow problems, labeled as types I, II, and III. When the respective theories are linearized, type I is similar to classical heat equation, whereas the linearized version of type II and type III theories allows propagation of thermal waves at finite speed. In type II and type III (i.e, thermoelasticity without energy dissipation and thermoelasticity with energy dissipation) the entropy flux vector is determined in terms of potential that also determines stresses. The temperature equation reduces to classical Fourier law of heat conduction when Fourier conductivity is dominant, and when the effect of conductivity is negligible, the equation has undamped thermal wave solutions without energy dissipation.

Various investigators have studied the different problems using GN type II and type III theories. Mukhopadhyay and Kumar [44] investigated interactions in an infinite medium with a cylindrical hole in generalized thermoelasticity. Mohamad et al. [45] studied Electromagneto thermoelastic 
problem in a thick plate using Green and Naghdi theory. Chitra and Ciarletta [46] studied harmonic vibrations in linear thermoelasticity without energy dissipation. Chitra and Ciarletta [47] proved several results in uniqueness and continuous dependence in thermoelasticity of type III. Passarella and Zampoli [48] proved reciprocal and variational principles in micropolar thermoelasticity of type II.

In the present investigation, we study the reflection of plane waves, that is, longitudinal displacement wave (LD wave), thermal wave (T-wave), and transverse wave coupled with microrotational wave (CD-I wave and CD-II wave) at the free surface of thermally conducting micropolar elastic medium with two temperatures with and without energy dissipation. Energy dissipation and two-temperature effects are depicted numerically and depicted graphically on the amplitude ratios for incidence of various plane waves for a particular model.

\section{Basic Equations}

Following Eringen (1970), Ezzat and Awad [17], and Green and Naghdi [42], the field equations in a micropolar thermoelastic medium with two temperatures, without body forces, body couples, and heat sources, are given by

$$
\begin{aligned}
& (\lambda+2 \mu+K) \nabla(\nabla \cdot \vec{u})-(\mu+K) \nabla \times(\nabla \times \vec{u}) \\
& +K(\nabla \times \vec{\phi})-\nu \nabla\left(1-a \nabla^{2}\right) \Phi=\rho \frac{\partial^{2} \vec{u}}{\partial t^{2}} \\
& (\alpha+\beta+\gamma) \nabla(\nabla \cdot \vec{\phi})-\gamma \nabla \times(\nabla \times \vec{\phi}) \\
& \quad+K \nabla \times \vec{u}-2 K \vec{\phi}=\rho j \frac{\partial^{2} \vec{\phi}}{\partial t^{2}}, \\
& K_{1} \nabla^{2} \Phi+K_{1}^{*} \nabla^{2} \dot{\Phi} \\
& =\rho c^{*} \frac{\partial^{2}}{\partial t^{2}}\left(1-a \nabla^{2}\right) \Phi+\nu T_{0} \frac{\partial^{2}}{\partial t^{2}}(\nabla \cdot \vec{u}),
\end{aligned}
$$

and the constitutive relations are

$$
\begin{gathered}
t_{i j}=\lambda u_{r, r} \delta_{i j}+\mu\left(u_{i, j}+u_{j, i}\right) \\
+K\left(u_{j, i}-\varepsilon_{i j r} \phi_{r}\right)-\nu T \delta_{i j}, \\
m_{i j}=\alpha \phi_{r, r} \delta_{i j}+\beta \phi_{i, j}+\gamma \phi_{j, i}, \quad i, j, r=1,2,3,
\end{gathered}
$$

where $\nabla^{2}$ is the Laplacian operator and $\lambda$ and $\mu$ are Lame's constants. $K, \alpha, \beta$, and $\gamma$ are micropolar constants. $t_{i j}$ and $m_{i j}$ are the components of stress tensor and couple stress tensor. $\vec{u}$ and $\vec{\phi}$ are the displacement and microrotation vectors, $\rho$ is the density, $\hat{j}$ is the microinertia, $K_{1}^{*}$ is the thermal conductivity, $K_{1}=c^{*}(\lambda+2 \mu) / 4$ is material characteristics constant of the theory, $c^{*}$ is the specific heat at constant strain, $\Phi$ is the deviation of conductive temperature from reference temperature, $T$ is the deviation of thermodynamic temperature from reference temperature, $T_{0}$ is the reference temperature, $\nu=(3 \lambda+2 \mu+K) \alpha_{T}$, where
$\alpha_{T}$ is the coefficient of linear thermal expansion, $\delta_{i j}$ is the Kronecker delta, and $\varepsilon_{i j r}$ is the alternate tensor. $T$ and $\Phi$ are connected by the relation $T=\left(1-a \nabla^{2}\right) \Phi$.

\section{Formulation of the Problem}

A homogeneous, isotropic, micropolar, thermoelastic solid half space with two temperatures (medium $M_{1}$ ) is considered. Origin of the rectangular Cartesian coordinate system $O x_{1} x_{2} x_{3}$ is taken on the surface $x_{3}=0$ and $x_{3}$-axis is pointing normally to the medium $M_{1}$.

The components of displacement and microrotation for two dimensional problem are taken as

$$
\begin{aligned}
& \vec{u}=\left(u_{1}\left(x_{1}, x_{3}\right), 0, u_{3}\left(x_{1}, x_{3}\right)\right), \\
& \vec{\phi}=\left(0, \phi_{2}\left(x_{1}, x_{3}\right), 0\right) .
\end{aligned}
$$

The dimensionless quantities are defined as

$$
\begin{aligned}
& x_{1}^{\prime}=\frac{x_{1}}{L}, \quad x_{3}^{\prime}=\frac{x_{3}}{L}, \quad u_{1}^{\prime}=\frac{u_{1}}{L}, \\
& u_{3}^{\prime}=\frac{u_{3}}{L}, \quad \phi_{2}^{\prime}=\frac{\lambda}{v T_{0}} \phi_{2}, \\
& t^{\prime}=\frac{c_{1}}{L} t, \quad T^{\prime}=\frac{T}{T_{0}}, \quad \Phi^{\prime}=\frac{\Phi}{T_{0}}, \\
& t_{i j}^{\prime}=\frac{1}{v T_{0}} t_{i j}, \quad m_{i j}^{\prime}=\frac{1}{L \nu T_{0}} m_{i j}, \quad a^{\prime}=\frac{1}{L} a,
\end{aligned}
$$

where $\omega^{*}=\rho c^{*} c_{1}^{2} / K^{*}, c_{1}^{2}=(\lambda+2 \mu+K) / \rho$, and $L$ is a parameter having dimensions of length.

The relations between nondimensional displacement components $u_{1}, u_{3}$ and the dimensionless potential functions $\phi, \psi$ can be expressed as

$$
u_{1}=\frac{\partial \phi}{\partial x_{1}}-\frac{\partial \psi}{\partial x_{3}}, \quad u_{3}=\frac{\partial \phi}{\partial x_{3}}+\frac{\partial \psi}{\partial x_{1}}
$$

Making use of (4) and (5) in (1) and with the aid of (6) after suppressing the primes, yields

$$
\begin{gathered}
a_{3} \nabla^{2} \phi-a_{2}\left(1-a \nabla^{2}\right) \Phi-a_{3} \frac{\partial^{2} \phi}{\partial t^{2}}=0 \\
\nabla^{2} \psi+a_{1} \phi_{2}-a_{3} \frac{\partial^{2} \psi}{\partial t^{2}}=0 \\
\nabla^{2} \phi_{2}-a_{4} \nabla^{2} \psi-2 a_{5} \phi_{2}-a_{6} \frac{\partial^{2} \phi_{2}}{\partial t^{2}}=0 \\
{\left[\nabla^{2}\left(1+a_{7} \frac{\partial}{\partial t}\right)-a_{8} \frac{\partial^{2}}{\partial t^{2}}\left(1-a \nabla^{2}\right)\right] \Phi-a_{9} \frac{\partial^{2}}{\partial t^{2}} \nabla^{2} \phi=0}
\end{gathered}
$$




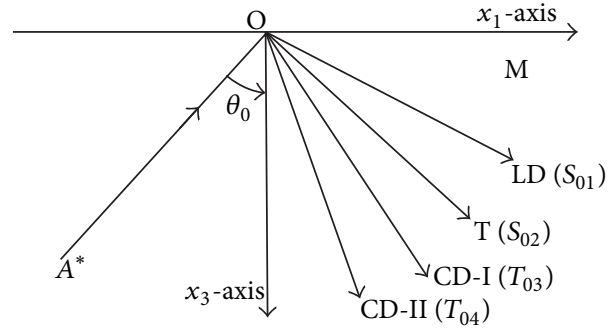

FIGURE 1: Geometry of the problem.

where

$$
\begin{aligned}
& a_{1}=\frac{K \nu T_{0}}{(\mu+K) \lambda}, \quad a_{2}=\frac{\nu T_{0}}{\mu+K}, \quad a_{3}=\frac{\rho c_{1}^{2}}{\mu+K}, \\
& a_{4}=\frac{K L^{2} \lambda}{\gamma \nu T_{0}}, \quad a_{5}=\frac{K L^{2}}{\gamma}, \quad a_{6}=\frac{\rho j c_{1}^{2}}{\gamma}, \\
& a_{7}=\frac{K_{1}^{*} c_{1}}{K_{1} L}, \quad a_{8}=\frac{\rho c^{*} c_{1}^{2}}{K_{1}}, \quad a_{9}=\frac{\gamma c_{1}^{2}}{K_{1}}, \\
& \nabla^{2}=\frac{\partial}{\partial x_{1}^{2}}+\frac{\partial}{\partial x_{3}^{2}} .
\end{aligned}
$$

\section{Boundary Conditions}

The following boundary conditions at the free surface $x_{3}=0$ are considered as

$$
\begin{aligned}
& t_{33}=0, \quad t_{31}=0 \\
& m_{32}=0, \quad\left(K_{1}+K_{1}^{*} \frac{\partial}{\partial t}\right) \frac{\partial T}{\partial x_{3}}=0 .
\end{aligned}
$$

\section{Reflection and Transmission}

We consider longitudinal displacement wave (LD-wave), thermal wave (T-wave), and coupled transverse and coupled microrotational waves (CD-I wave and CD-II wave) propagating through micropolar thermoelastic with twotemperature solid half space $x_{3}>0$ and incident at the plane $x_{3}=0$ with its direction of propagation making an angle $\theta_{0}$ normal to the surface. Corresponding to each incident wave, we get reflected LD-wave, T-wave, CD-I, and CD-II waves as shown in Figure 1.

In order to solve (7), we assume the solutions of the form

$$
\left\{\phi, \Phi, \psi, \phi_{2}\right\}=\left\{\widetilde{\phi}, \widetilde{\Phi}, \widetilde{\psi}, \widetilde{\phi}_{2}\right\} e^{l\left\{k\left(x_{1} \sin \theta-x_{3} \cos \theta\right)-\omega t\right\}},
$$

where $k$ is the wave number, $\omega$ is the angular frequency, and $\widetilde{\phi}, \widetilde{\Phi}, \widetilde{\psi}$, and $\widetilde{\phi}_{2}$ are arbitrary constants.

Using (10) in (7), we obtain

$$
\begin{aligned}
& V^{4}+D_{1} V^{2}+E_{1}=0, \\
& V^{4}+D_{2} V^{2}+E_{2}=0,
\end{aligned}
$$

where

$$
\begin{aligned}
& D_{1}=\frac{-a_{3} a_{8}\left(1-a \omega^{2}\right)+i \omega a_{3}\left((i / \omega)+a_{7}\right)-a_{2} a_{9}}{a_{3} a_{8}}, \\
& E_{1}=-\frac{a_{3} i \omega\left((i / \omega)+a_{7}\right)+a\left(a_{3} a_{8}+a_{2} a_{9}\right)}{a_{3} a_{8}}, \\
& D_{2}=-\left(\frac{a_{1} a_{4}}{\omega^{2}}+a_{3}\right) \frac{1}{a_{3}\left(a_{6}-\left(2 a_{5} / \omega^{2}\right)\right)}-\frac{1}{a_{3}}, \\
& E_{2}=\frac{1}{\left(a_{6}-\left(2 a_{5} / \omega^{2}\right)\right) a_{3}}, \quad V^{2}=\frac{\omega^{2}}{k^{2}} .
\end{aligned}
$$

Equations (11) and (12) are quadratic in $V^{2}$; therefore the roots of these equations give four values of $V^{2}$. Corresponding to each value of $V^{2}$ in (11), there exist two types of waves in decreasing order of their velocities, namely, LD-wave and Twave. Similarly corresponding to each value of $V^{2}$ in (12), there exist two types of waves, namely, CD-I wave and CDII wave. Let $V_{1}, V_{2}$ be the velocities of reflected LD-wave, T-wave and $V_{3}, V_{4}$ the velocities of reflected CD-I wave, CDII wave.

In view of (10), the appropriate solutions of (7) are assumed of the form

$$
\begin{gathered}
\{\phi, \Phi\}=\sum_{i=1}^{2}\left\{1, f_{i}\right\}\left[S_{0 i} e^{\ell\left\{k_{i}\left(x_{1} \sin \theta_{0 i}-x_{3} \cos \theta_{0 i}\right)-\omega_{i} t\right\}}+P_{i}\right], \\
\left\{\psi, \phi_{2}\right\}=\sum_{j=3}^{4}\left\{1, f_{j}\right\}\left[T_{0 j} e^{l\left\{k_{j}\left(x_{1} \sin \theta_{0 j}-x_{3} \cos \theta_{0 j}\right)-\omega_{j} t\right\}}+P_{j}\right],
\end{gathered}
$$

where

$$
\begin{aligned}
& f_{i}=\frac{-\iota \omega_{i}\left[\left(a_{2} a_{9} / V_{i}^{2}\right)+a_{3} a_{8}\left(\left(1 / V_{i}^{2}\right)-1\right)\right]}{a_{2} / V_{i}^{2}\left(\left(\iota / \omega_{i}\right)+a_{7}\right)}, \\
& f_{j}=\frac{a_{3} a_{4}}{\left(1 / V_{j}^{2}\right)+\left(\left(2 a_{5}-a_{1} a_{4}\right) / \omega_{j}^{2}\right)-a_{6}}, \\
& P_{i}=S_{i} e^{\left\{k_{i}\left(x_{1} \sin \theta_{i}+x_{3} \cos \theta_{i}\right)-\omega_{i} t\right\}}, \\
& P_{j}=T_{j} e^{\left\lfloor\left\{k_{j}\left(x_{1} \sin \theta_{j}+x_{3} \cos \theta_{j}\right)-\omega_{j} t\right\}\right.},
\end{aligned}
$$

and $S_{0 i}, T_{0 j}$ are the amplitudes of incident LD-wave, Twave and CD-I, CD-II waves, respectively. $S_{i}$ and $T_{j}$ are the amplitudes of reflected LD-wave, T-wave and CD-I, CD-II waves, respectively.

We use the following extension of Snell's law to satisfy the boundary conditions:

$$
\frac{\sin \theta_{0}}{V_{0}}=\frac{\sin \theta_{1}}{V_{1}}=\frac{\sin \theta_{2}}{V_{2}}=\frac{\sin \theta_{3}}{V_{3}}=\frac{\sin \theta_{4}}{V_{4}},
$$

where

$$
V_{j}=\frac{\omega}{k_{j}}, \quad(j=1,2,3,4) \text { at } x_{3}=0 .
$$


Making use of the values of $\phi, \psi, \Phi$, and $\phi_{2}$ from (14) in boundary conditions (9) and with the aid of (2)-(6), (16), and (17), we obtain a system of four nonhomogeneous equations in the following form:

$$
\sum_{j=1}^{4} a_{i j} Z_{j}=Y_{i} ; \quad(i=1,2,3,4),
$$

where the values of $a_{i j}$ are given as

$$
\begin{aligned}
& a_{1 i}=\left(d_{1}+d_{2} B_{i}\right) \frac{\omega^{2}}{V_{i}^{2}}+\left(1+a \frac{\omega^{2}}{V_{i}^{2}}\right) f_{i}, \\
& a_{1 j}=d_{2} \frac{\omega^{2}}{V_{j} V_{0}} \sin \theta_{0} \sqrt{B_{j}} \text {, } \\
& a_{2 i}=-\left(2 d_{3}+d_{4}\right) \frac{\omega^{2}}{V_{1} V_{0}} \sin \theta_{0} \sqrt{B_{i}} \text {, } \\
& a_{2 j}=\left(d_{3}+d_{4}\right) \frac{\omega^{2}}{V_{j}^{2}} B_{j}-d_{3} \frac{\omega^{2}}{V_{0}^{2}} \sin ^{2} \theta_{0}-d_{4} f_{j} \text {, } \\
& a_{3 i}=0, \quad a_{3 j}=\iota \frac{\omega}{V_{j}} \sqrt{B_{j}} f_{j}, \\
& a_{4 i}=\iota \frac{\omega}{V_{i}}\left(1+a \frac{\omega^{2}}{V_{i}^{2}}\right) f_{i} \sqrt{B_{i}}, \quad a_{4 j}=0 \\
& (i=1,2, \quad j=3,4), \\
& d_{1}=\frac{\lambda}{v T_{0}}, \quad d_{2}=\frac{(2 \mu+K)}{v T_{0}}, \\
& d_{3}=\frac{\mu}{v T_{0}}, \quad d_{4}=\frac{K}{v T_{0}}, \quad \bar{p}_{0}=\frac{\bar{\nu}}{\nu}, \\
& B_{i}=\left(1-\frac{V_{i}^{2}}{V_{0}^{2}} \sin ^{2} \theta_{0}\right), \quad B_{j}=\left(1-\frac{V_{j}^{2}}{V_{0}^{2}} \sin ^{2} \theta_{0}\right), \\
& R_{i}=\left(1-\frac{\bar{V}_{i}^{2}}{V_{0}^{2}} \sin ^{2} \theta_{0}\right), \quad R_{j}=\left(1-\frac{\bar{V}_{j}^{2}}{V_{0}^{2}} \sin ^{2} \theta_{0}\right) \\
& (i=1,2, \quad j=3,4, \quad k=5,6, \quad l=7,8),
\end{aligned}
$$

and also

$$
\begin{array}{ll}
Z_{1}=\frac{S_{1}}{A^{*}}, & Z_{2}=\frac{S_{2}}{A^{*}}, \\
Z_{3}=\frac{T_{3}}{A^{*}}, & Z_{4}=\frac{T_{4}}{A^{*}},
\end{array}
$$

where $Z_{1}, Z_{2}, Z_{3}$, and $Z_{4}$ are the amplitude ratios of reflected LD-wave, T-wave and coupled CD-I, CD-II waves.

(1) For incident LD-wave:

$$
\begin{aligned}
& A^{*}=S_{01}, \quad S_{02}=T_{03}=T_{04}=0, \quad Y_{1}=-a_{11}, \\
& Y_{2}=a_{21}, \quad Y_{3}=a_{31}=0, \quad Y_{4}=a_{41} .
\end{aligned}
$$

(2) For incident T-wave:

$$
\begin{aligned}
& A^{*}=S_{02}, \quad S_{01}=T_{03}=T_{04}=0, \\
& Y_{1}=-a_{12}, \quad Y_{2}=a_{22}, \quad Y_{3}=a_{32}=0, \quad Y_{4}=a_{42} .
\end{aligned}
$$

(3) For incident CD-I wave:

$$
\begin{array}{ll}
A^{*}=T_{03}, & S_{01}=S_{02}=T_{04}=0, \\
Y_{1}=a_{13}, & Y_{2}=-a_{23}, \quad Y_{3}=a_{33}, \quad Y_{4}=a_{43}=0 .
\end{array}
$$

(4) For incident CD-II wave:

$$
\begin{array}{ll}
A^{*}=T_{04}, & S_{01}=S_{02}=T_{03}=0, \quad Y_{1}=a_{14}, \\
Y_{2}=-a_{24}, & Y_{3}=a_{34}, \quad Y_{4}=a_{44}=0 .
\end{array}
$$

5.1. Medium Reduced to Micropolar Thermoelastic with Energy Dissipation and without Two Temperatures (ATS). By neglecting two-temperature parameters, that is, $a \rightarrow 0$ in (18), we obtain the amplitude ratios at the free surface of micropolar thermoelastic solid half space with energy dissipation with changed values of $a_{1 i}$ and $a_{4 i}(i=1,2)$ as

$$
\begin{array}{r}
a_{1 i}=\left(d_{1}+d_{2} B_{i}\right) \frac{\omega^{2}}{V_{i}^{2}}+f_{i}, \quad a_{4 i}=\iota \frac{\omega}{V_{i}} f_{i} \sqrt{B_{i}}, \\
(i=1,2)
\end{array}
$$

and the remaining entries remain the same.

5.2. Medium Reduced to Micropolar Thermoelastic with Two Temperatures and without Energy Dissipation (KTS). If we take $K_{1}^{*} \rightarrow 0$ in (18), then we obtain the amplitude ratios at the free surface of micropolar thermoelastic solid half space with two temperatures and without energy dissipation. The values of $D_{1}, D_{2}, E_{1}$, and $E_{2}$ in (13) take the form

$$
\begin{aligned}
& D_{1}=\frac{-a_{3} a_{8}\left(1-a \omega^{2}\right)-a_{3}-a_{2} a_{9}}{a_{3} a_{8}}, \\
& E_{1}=-\frac{-a_{3} i \omega+a\left(a_{3} a_{8}+a_{2} a_{9}\right)}{a_{3} a_{8}}, \\
& D_{2}=-\left(\frac{a_{1} a_{4}}{\omega^{2}}+a_{3}\right) \frac{1}{a_{3}\left(a_{6}-\left(2 a_{5} / \omega^{2}\right)\right)}-\frac{1}{a_{3}}, \\
& E_{2}=\frac{1}{\left(a_{6}-\left(2 a_{5} / \omega^{2}\right)\right) a_{3}} .
\end{aligned}
$$

5.3. Medium Reduced to Thermoelastic without Energy Dissipation and without Two Temperatures (AKTS). If we take $K_{1}^{*} \rightarrow 0, a \rightarrow 0$ in (18), then we obtain the amplitude ratios at the free surface of micropolar thermoelastic solid half space without energy dissipation. The values of $a_{i j}, D_{1}$, $D_{2}, E_{1}$, and $E_{2}$ are given by (25) and (26). 


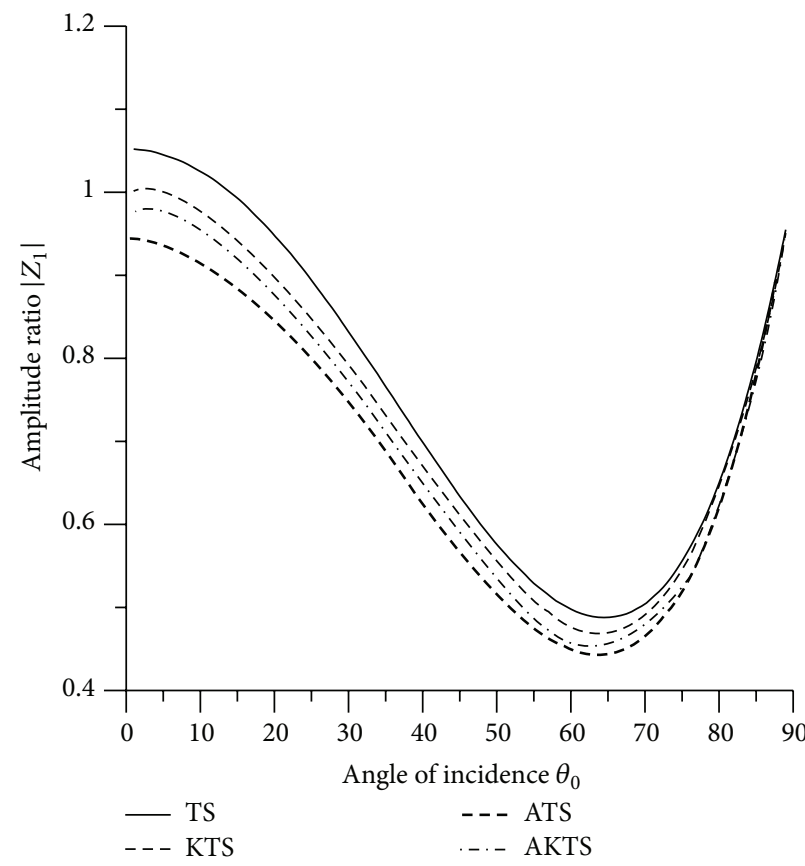

(a)

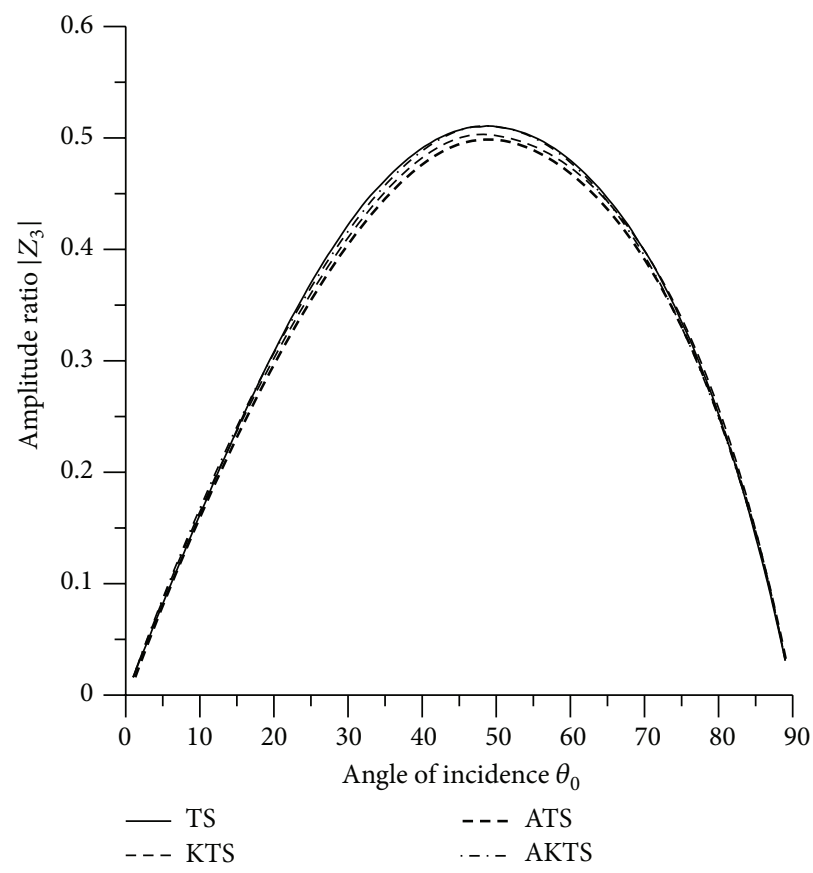

(c)

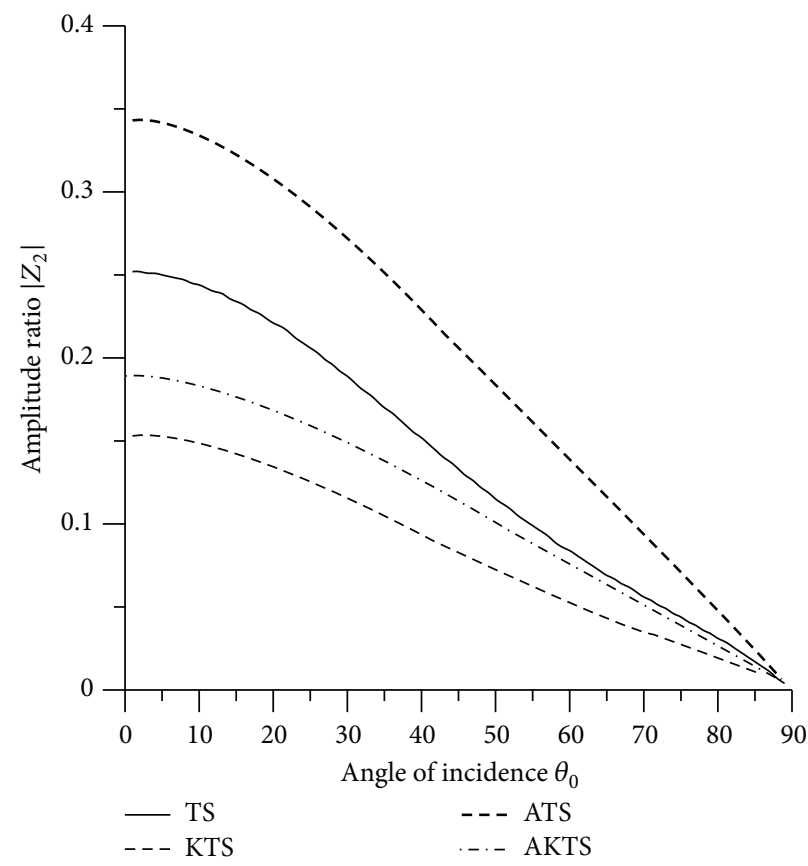

(b)

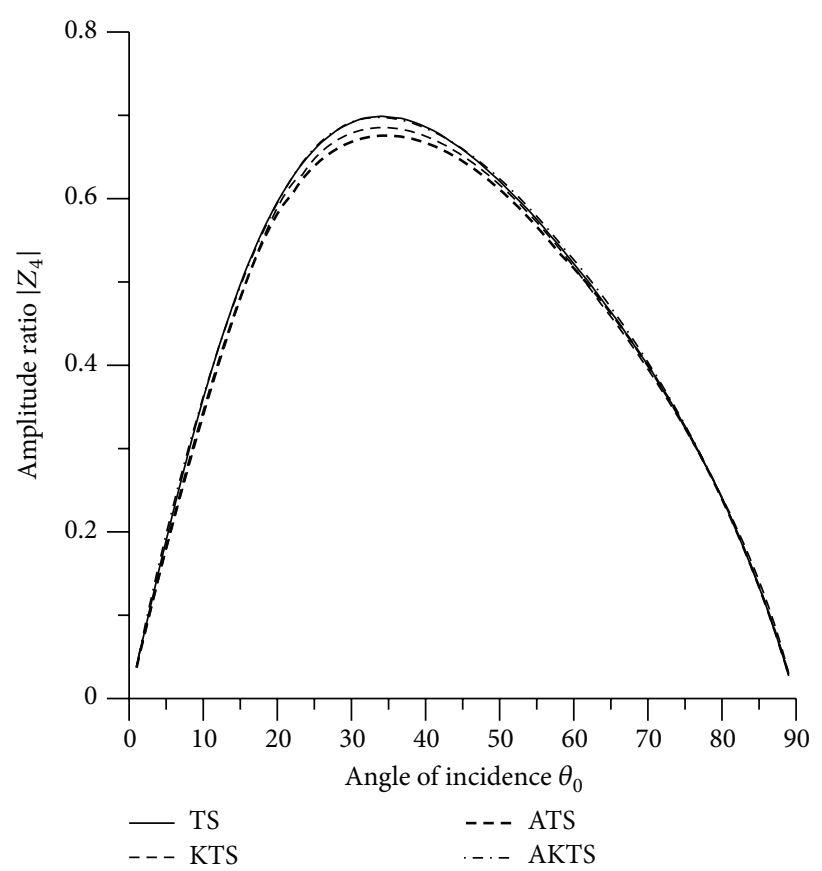

(d)

FIGURE 2: Variations of amplitude ratios with angle of incidence for LD-wave.

\section{Numerical Results and Discussion}

The following values of relevant parameters for numerical computations are taken.

Following Eringen [49] the values of micropolar constants are taken as

$$
\begin{array}{ll}
\lambda=9.4 \times 10^{10} \mathrm{Nm}^{-2}, & \mu=4.0 \times 10^{10} \mathrm{Nm}^{-2}, \\
K=1.0 \times 10^{10} \mathrm{Nm}^{-2}, & \gamma=7.79 \times 10^{-10} \mathrm{~N},
\end{array}
$$

$$
\widehat{j}=0.002 \times 10^{-17} \mathrm{~m}^{2}, \quad \rho=1.74 \times 10^{3} \mathrm{Kgm}^{-3} .
$$

Following Dhaliwal and Singh [50] the values of thermal parameters are taken as

$$
\begin{aligned}
& v=2.68 \times 10^{4} \mathrm{Nm}^{-2} \mathrm{~K}^{-1}, \\
& c^{*}=1.04 \times 10^{3} \mathrm{~m}^{2} \mathrm{sec}^{-2} \mathrm{~K}^{-1}, \quad a=0.5 \mathrm{~m}^{2},
\end{aligned}
$$




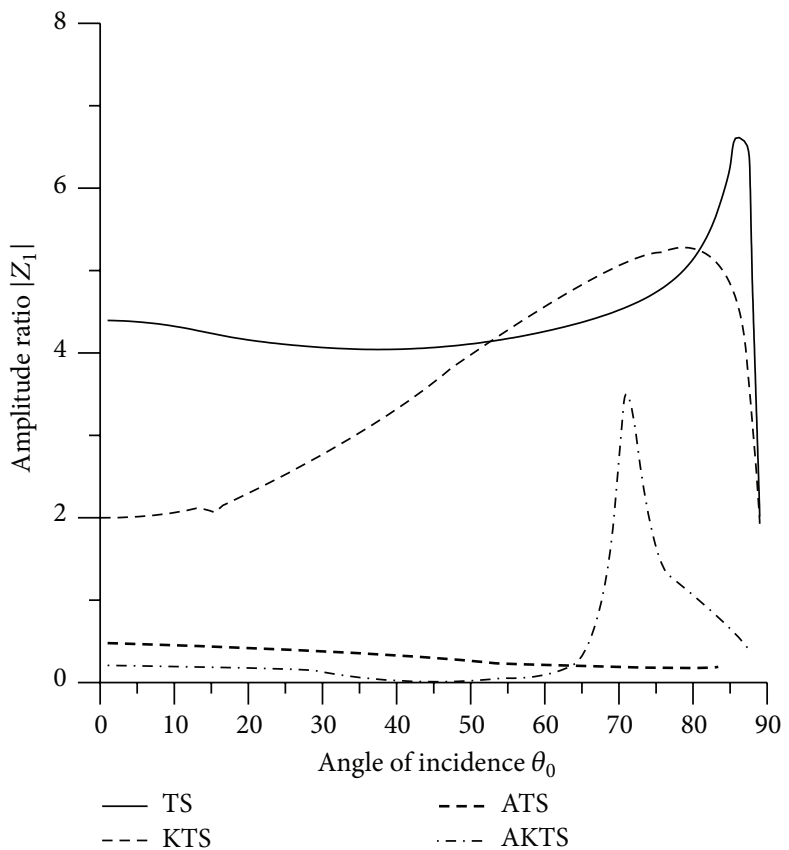

(a)

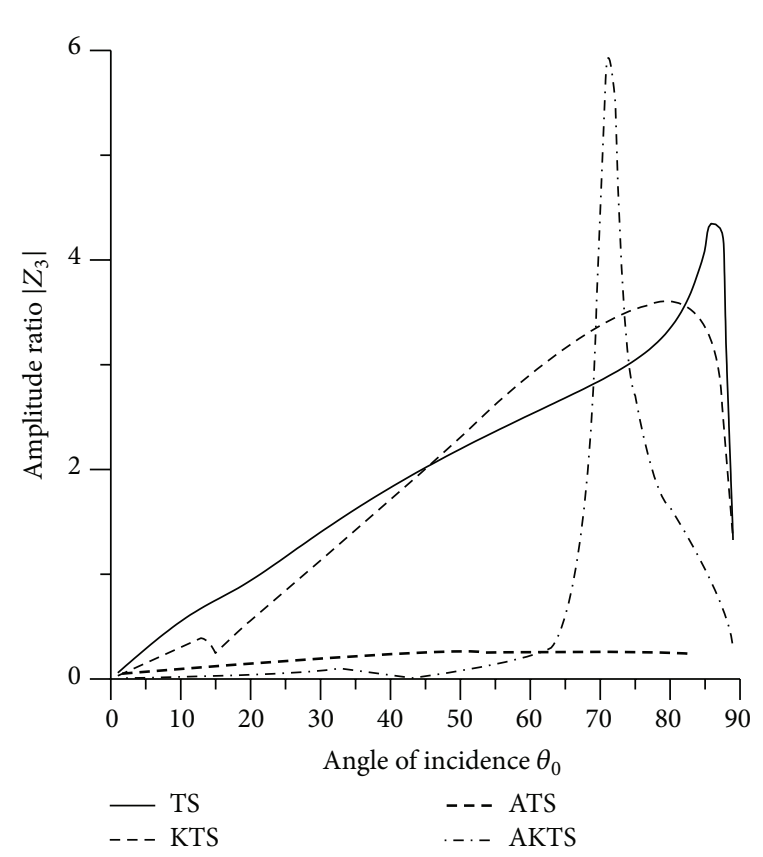

(c)

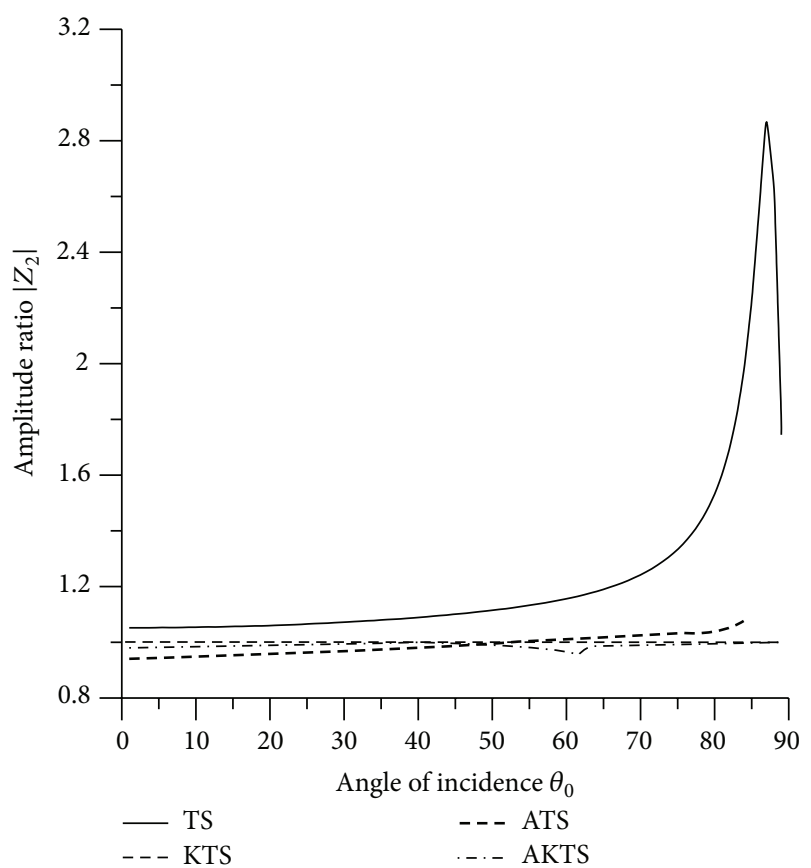

(b)

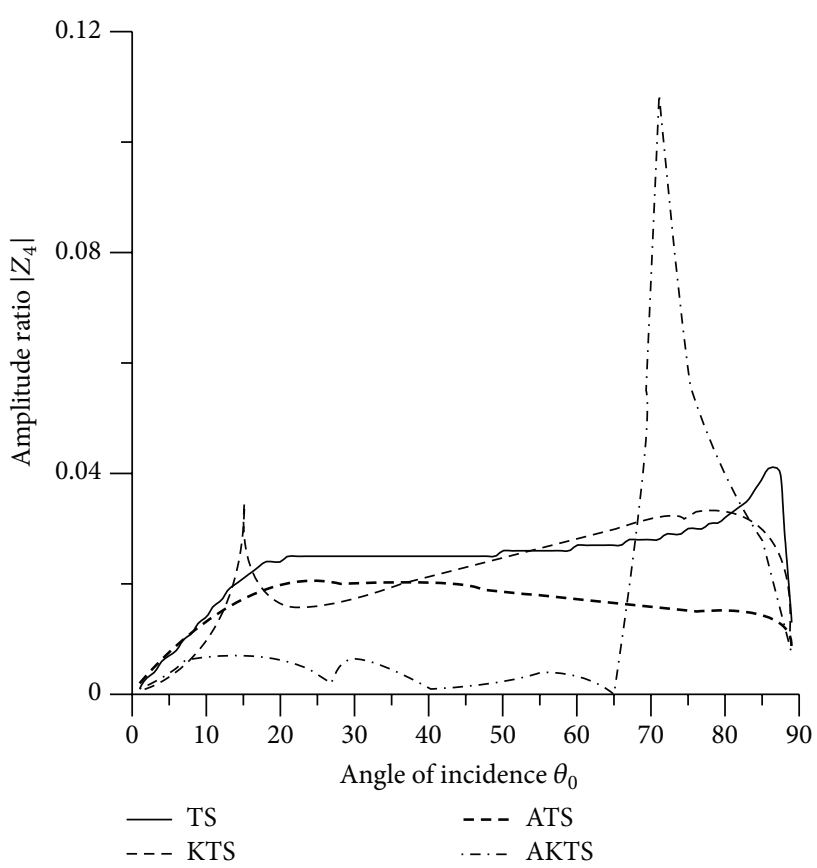

(d)

FIGURE 3: Variations of amplitude ratios with angle of incidence for T-wave.

$T_{0}=0.298 \mathrm{~K}, \quad K_{1}^{*}=1.7 \times 10^{2} \mathrm{Nsec}^{-1} \mathrm{~K}^{-1}, \quad \omega=1$.

The values of amplitude ratios have been computed at different angles of incidence.

In Figures 2(a)-4(d), we represent the solid line for incident wave for thermoelastic solid with two temperatures and with energy dissipation (TS), small dashe line for thermoelastic solid with two temperatures and without energy dissipation (KTS), large dashed line for thermoelastic solid with energy dissipation (ATS), and dashed dot dashed line for thermoelastic solid without energy dissipation (AKTS).

6.1. Incident $L D$-Wave. Variations of amplitude ratios $\left|Z_{i}\right|$, $1 \leq i \leq 4$, with the angle of incidence $\theta_{0}$, for incident LDwave, are shown in Figures 2(a)-2(d). 


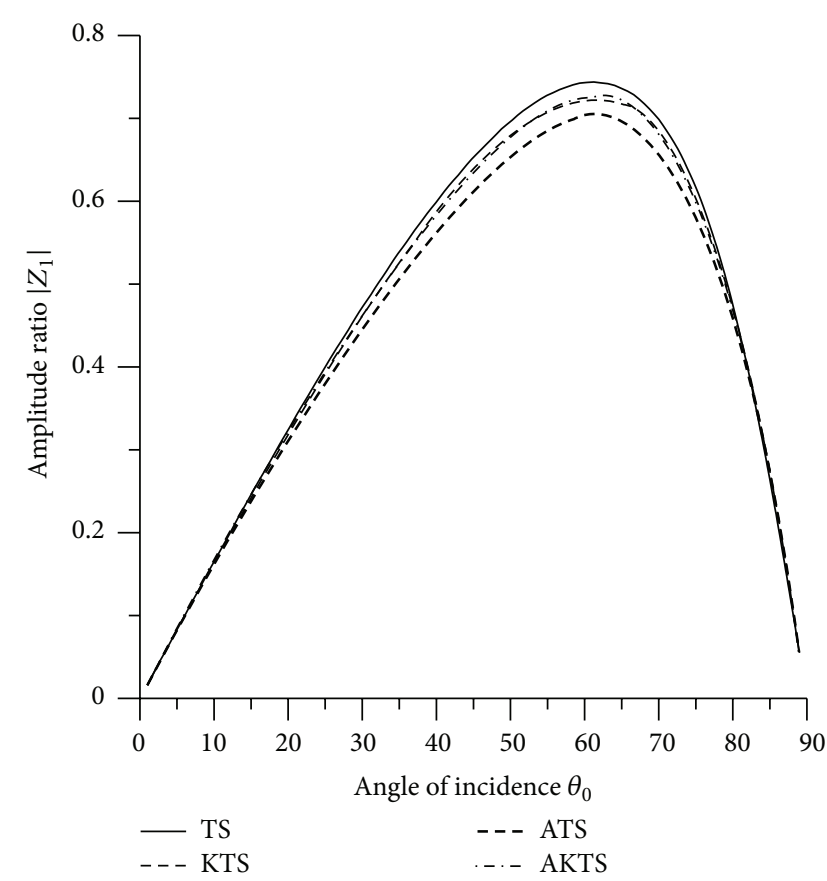

(a)

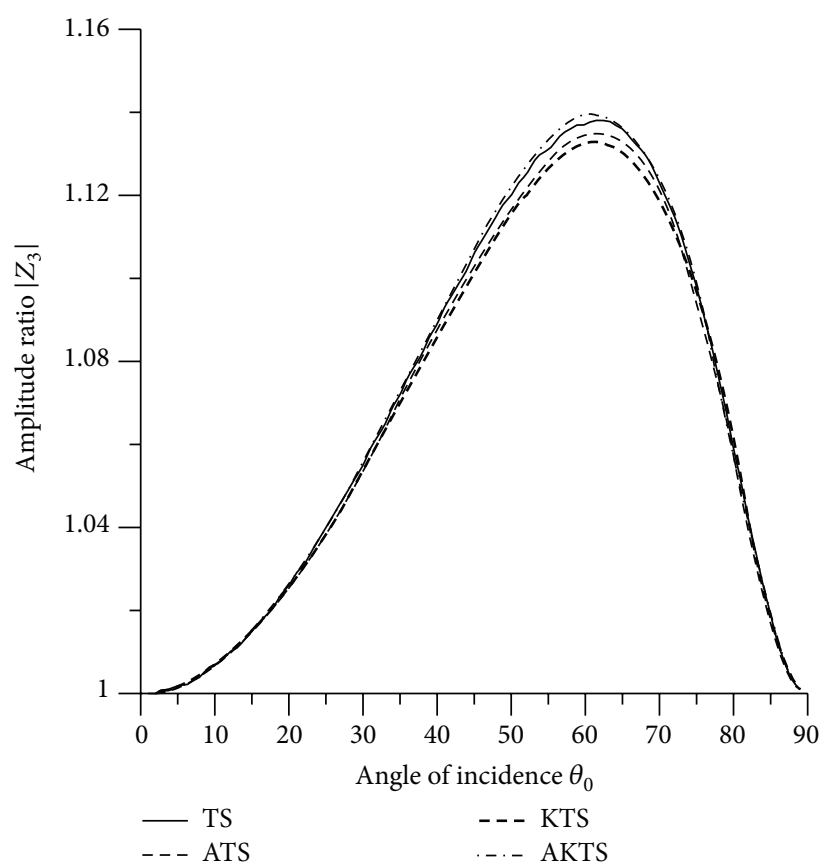

(c)

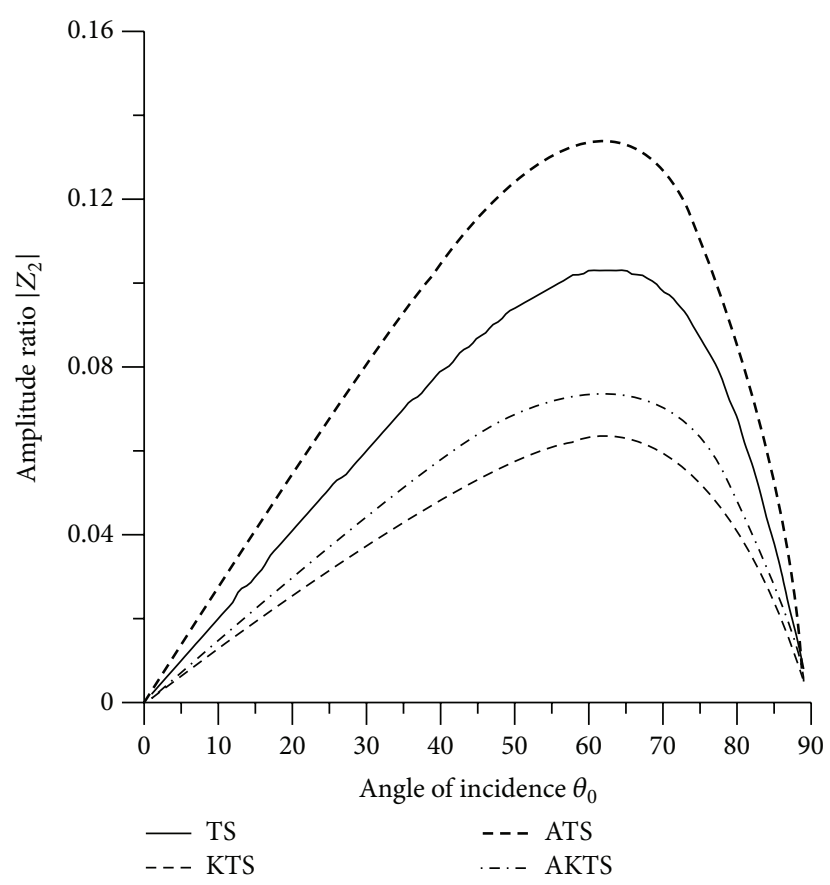

(b)

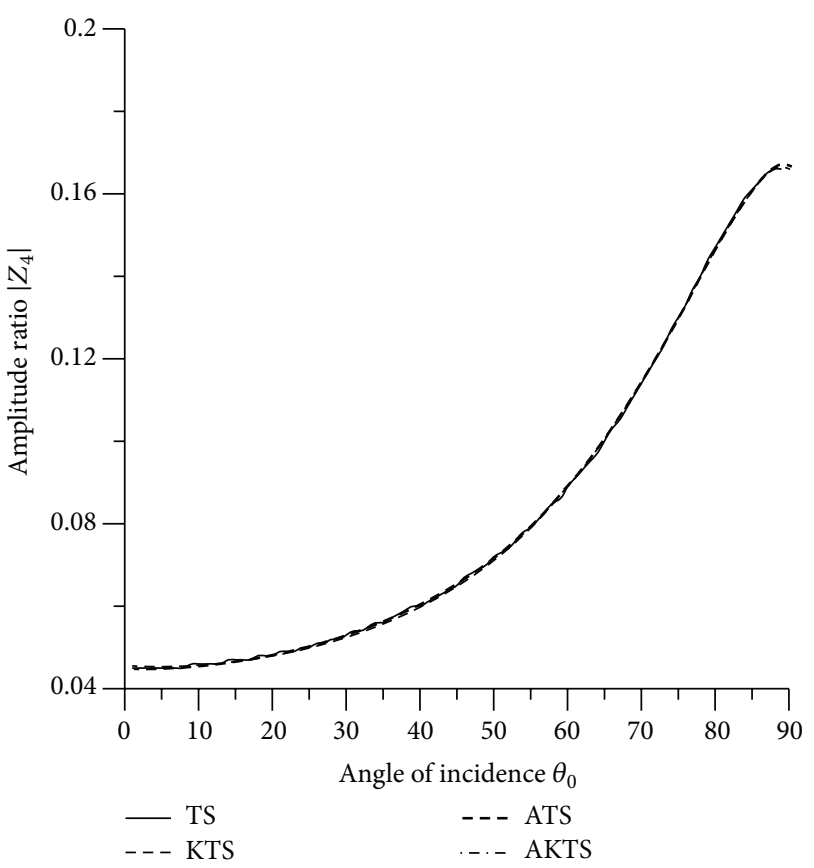

(d)

FIGURE 4: Variations of amplitude ratios with angle of incidence for CD-I wave.

Figure 2(a) shows that the values of $\left|Z_{1}\right|$ for TS, KTS, ATS, and AKTS are oscillatory in the whole range. The values for TS attain maximum value near the normal incidence. The value of $\left|Z_{1}\right|$ decreases monotonically attaining the minimum value at $\theta_{0}=65^{\circ}$, and then increases for all the cases. Also the values for TS in comparison with KTS and AKTS in comparison with ATS remain greater in the whole range.
It is evident from Figure 2(b) that the values of amplitude ratio $\left|Z_{2}\right|$ for TS, KTS, ATS, and AKTS decrease in the whole range. Also the values for ATS in comparison with TS and AKTS in comparison with KTS remain more in the whole range. The values of $\left|Z_{2}\right|$ attain the maximum value at the normal incidence and then decrease rapidly and converge to the grazing incidence. The values of amplitude ratio for TS, KTS, ATS, and AKTS are magnified by multiplying by 10 . 
Figure 2(c) shows that the values for $\left|Z_{3}\right|$ for TS, KTS, ATS, and AKTS increase up to intermediate range and then decrease with further increase in angle of incidence. The values for TS remain greater than the values for KS, ATS, and AKTS in the whole range. The values of $\left|Z_{3}\right|$ attain the minimum value at normal and grazing incidences and attain the maximum at $\theta_{0}=50^{\circ}$.

Figure 2(d) depicts that the values of amplitude ratio $\left|Z_{4}\right|$ for TS, KTS, ATS, and AKTS increase in the range $0^{\circ}<\theta_{0}<$ $32^{\circ}$ and then decrease in the subsequent range. The values of $\left|Z_{4}\right|$ depict the similar behavior as $\left|Z_{3}\right|$ with difference in their magnitude values. The values of amplitude ratio for TS, KTS, ATS, and AKTS are magnified by multiplying by $10^{2}$.

6.2. Incident $T$-Wave. Variations of amplitude ratios $\left|Z_{i}\right|, 1 \leq$ $i \leq 4$, with the angle of incidence $\theta_{0}$, for incident T-wave, are shown in Figures 3(a)-3(d).

Figure 3(a) shows that the values of $\left|Z_{1}\right|$ for TS, KTS, ATS, and AKTS oscillate in the whole range. The values for AKTS attain peak value in the interval $65^{\circ}<\theta_{0}<75^{\circ}$. The values for TS are demagnified by dividing by 10 .

Figure 3(b) depicts that the amplitude of $\left|Z_{2}\right|$ for TS increases in the whole range, except near the grazing incidence where it decreases. Also the values for KTS in comparison with AKTS and TS in comparison with ATS remain more in the whole range that shows the effect of two temperatures. Similar behavior of $\left|Z_{2}\right|$ is noticed for all the cases except TS.

It is evident from Figure 3(c) that the values of $\left|Z_{3}\right|$ for AKTS attain maximum value in the interval $70^{\circ}<\theta_{0}<80^{\circ}$. The values for TS and ATS increase in the whole range, except near the grazing incidence where the values decrease.

Figure 3(d) depicts that values of $\left|Z_{4}\right|$ for TS in comparison with ATS are greater. The values of amplitude ratio for KTS and AKTS attain peak value in the ranges $10^{\circ}<\theta_{0}<20^{\circ}$ and $70^{\circ}<\theta_{0}<80^{\circ}$, respectively. The values of amplitude ratio for TS, KTS, and AKTS are magnified by multiplying by 10 and the values for ATS are magnified by multiplying by $10^{2}$.

6.3. Incident $C D-I$ Wave. Variations of amplitude ratios $\left|Z_{i}\right|, 1 \leq i \leq 4$, with the angle of incidence $\theta_{0}$, for incident CD-I wave, are shown in Figures 4(a)-4(d).

Figure 4(a) depicts that the values of $\left|Z_{1}\right|$ for TS, KTS, ATS, and AKTS increase in the interval $0^{\circ}<\theta_{0}<66^{\circ}$ and then decrease with increase in $\theta_{0}$. Also the values for TS are greater than the values for KTS, ATS, and AKTS in the whole range. The behavior and variation of $\left|Z_{1}\right|$ near the normal incidence and grazing incidences are similar for all the considered cases.

It is depicted from Figure 4(b) that the values of $\left|Z_{2}\right|$ for AKTS in comparison with KTS are greater in the whole range. The maximum value is attained by ATS in the interval $55^{\circ}<\theta_{0}<66^{\circ}$. The trend of variation of $\left|Z_{2}\right|$ for all the cases at the normal and grazing incidence is similar. The values of amplitude ratio for TS, KTS, and AKTS are magnified by multiplying by $10^{2}$ and the values for ATS are magnified by multiplying by 10 .

It is noticed from Figure 4(c) that the values of $\left|Z_{3}\right|$ for TS, KTS, ATS, and AKTS first increase and then get decreased.
Also the values for AKTS are higher than the values for KTS in the whole range. It is evident that at the normal and grazing incidences the value of $\left|Z_{3}\right|$ is the same.

Figure 4(d) depicts that the values of $\left|Z_{4}\right|$ for TS, ATS, KTS, and AKTS increase in the whole range. The maximum value is attained by TS at the grazing incidence. Also there is slight difference in the magnitudes for TS, ATS, KTS, and AKTS.

\section{Conclusion}

In the present paper, the expressions for reflection coefficients of various reflected waves have been derived in micropolar thermoelastic solid half space in the context of GN type II and GN type III theories. It is observed that when LD-wave is incident, the values of amplitude ratios for TS remain more than the value for ATS; that is, two-temperature effect increases the magnitude of amplitude ratios. Also when T-wave is incident, the values of amplitude ratios follow oscillatory pattern and the values for KTS and AKTS attain peak value near the grazing incidence. The values of amplitude ratio $\left|Z_{i}\right|, 1 \leq$ $i \leq 4$, for TS in comparison with KTS remain greater which reveals the effect of energy dissipation (CD-I wave incident). The problem is of geophysical interest and the results are supposed to be useful in theoretical and observational studies of wave propagation in more realistic models of micropolar solids present in the earth's interior.

\section{Conflict of Interests}

The authors declare that there is no conflict of interests regarding the publication of this paper.

\section{References}

[1] A. C. Eringen, "Linear Theory of micropolar elasticity," Journal of Applied Mathematics and Mechanics, vol. 15, pp. 909-923, 1966.

[2] A. C. Eringen, Foundations of Micropolar Thermoelasticity, Udline Course and Lectures 23, International Centre for Mechanical Science, Springer, Berlin, Germany, 1970.

[3] A. C. Eringen, Microcontinuum Field Theories-I, Foundations and Solids, Springer, Berlin, Germany, 1999.

[4] W. Nowacki, Theory of Asymmetric Elasticity, Pergamon, Oxford, UK, 1986.

[5] S. Dost and B. Tabarrok, "Generalized micropolar thermoelasticity," International Journal of Engineering Science, vol. 16, no. 3, pp. 173-183, 1978.

[6] D. S. Chandrasekharaiah, "Heat-flux dependent micropolar thermoelasticity," International Journal of Engineering Science, vol. 24, no. 8, pp. 1389-1395, 1986.

[7] E. Boschi and D. Ieşan, "A generalized theory of linear micropolar thermoelasticity," Meccanica, vol. 8, no. 3, pp. 154-157, 1973.

[8] P. J. Chen and W. O. Williams, "A note on non-simple heat conduction," Zeitschrift für Angewandte Mathematik und Physik, vol. 19, no. 6, pp. 969-970, 1968.

[9] P. J. Chen, M. E. Gurtin, and W. O. Williams, "On the thermodynamics of non-simple elastic materials with two temperatures," Zeitschrift für Angewandte Mathematik und Physik, vol. 20, no. 1, pp. 107-112, 1969. 
[10] B. A. Boley and I. S. Tolins, "Transient coupled thermoelastic boundary value problem in the half space," Journal of Applied Mechanics, vol. 29, pp. 637-646, 1962.

[11] W. E. Warren and P. J. Chen, "Wave propagation in the two temperature theory of thermoelasticity," Acta Mechanica, vol. 16, no. 1-2, pp. 21-33, 1973.

[12] H. M. Youssef, "Theory of two-temperature-generalized thermoelasticity," IMA Journal of Applied Mathematics, vol. 71, no. 3, pp. 383-390, 2006.

[13] H. M. Youssef and E. Bassiouny, "Two temperature generalized thermopiezoelasticity for one dimensional problemsstate space approach," Computational Methods in Science and Technology, vol. 14, pp. 155-164, 2008.

[14] E. Bassiouny and H. M. Youssef, "Two-temperature generalized thermopiezoelasticity of finite rod subjected to different types of thermal loading," Journal of Thermal Stresses, vol. 31, no. 3, pp. 233-245, 2008.

[15] M. A. Ezzat and A. A. Bary, "State space approach of twotemperature magneto-thermoelasticity with thermal relaxation in a medium of perfect conductivity," International Journal of Engineering Science, vol. 47, no. 4, pp. 618-630, 2009.

[16] R. Kumar, R. Prasad, and S. Mukhopadhyay, "Variational and reciprocal principles in two-temperature generalized thermoelasticity," Journal of Thermal Stresses, vol. 33, no. 3, pp. 161-171, 2010.

[17] M. A. Ezzat and E. S. Awad, "Constitutive relations, uniqueness of solution, and thermal shock application in the linear theory of micropolar generalized thermoelasticity involving two temperatures," Journal of Thermal Stresses, vol. 33, no. 3, pp. 226250, 2010 .

[18] R. Kumar and S. Mukhopadhyay, "Effects of thermal relaxation time on plane wave propagation under two-temperature thermoelasticity," International Journal of Engineering Science, vol. 48, no. 2, pp. 128-139, 2010.

[19] S. Kaushal, N. Sharma, and R. Kumar, "Propagation of waves in generalized thermoelastic continua with two temperature," International Journal of Applied Mechanics and Engineering, vol. 15, pp. 1111-1127, 2010.

[20] S. Kaushal, R. Kumar, and A. Miglani, "Wave propagation in temperature rate dependent thermoelasticity with two temperatures," Mathematical Sciences, vol. 5, pp. 125-146, 2011.

[21] S. S. Shaw and B. Mukhopadhyay, "Generalized theory of micropolar-fractional-ordered thermoelasticity with two temperature," Journal of Applied Mathematics and Mechanics, vol. 7, pp. 32-48, 2011.

[22] H. M. Youssef, "Theory of two-temperature thermoelasticity without energy dissipation," Journal of Thermal Stresses, vol. 34, no. 2, pp. 138-146, 2011.

[23] A. S. El-Karamany, "Two-temperature theory in linear micropolar thermoviscoelastic anisotropic solid," Journal of Thermal Stresses, vol. 34, no. 9, pp. 985-1000, 2011.

[24] A. S. El-Karamany and M. A. Ezzat, "On the two-temperature Green-Naghdi thermoelasticity theories," Journal of Thermal Stresses, vol. 34, no. 12, pp. 1207-1226, 2011.

[25] S. Mukhopadhyay, R. Prasad, and R. Kumar, "On the theory of two-temperature thermoelasticity with two phase-lags," Journal of Thermal Stresses, vol. 34, no. 4, pp. 352-365, 2011.

[26] M. A. Ezzat, El-Karamany, and S. M. Ezzat, "Two-temperature theory in magneto-thermoelasticity with fractional order dualphase-lag heat transfer," Nuclear Engineering and Design, vol. 252, pp. 267-277, 2012.
[27] B. Singh and K. Bala, "Reflection of P and SV waves from the free surface of a two-temperature thermoelastic solid halfspace," Journal of Mechanics of Material and Structure, vol. 7, pp. 183-193, 2012.

[28] H. M. Youssef, "Variational principal of two temperature thermoelasticity without energy dissipation," Journal of Thermoelasticity, vol. 1, pp. 42-44, 2013.

[29] S. Banik and M. Kanoria, "Study of two temperature generalized thermopiezoelastic problem," Journal of Thermal Stresses, vol. 36, pp. 71-93, 2013.

[30] M. A. Ezzat and M. I. Othman, "Electromagneto-thermoelastic plane waves with two relaxation times in a medium of perfect conductivity," International Journal of Engineering Science, vol. 38, no. 1, pp. 107-120, 2000.

[31] M. A. Ezzat, M. I. Othman, and A. S. El-Karamany, "Electromagneto-thermoelastic plane waves with thermal relaxation in a medium of perfect conductivity," Journal of Thermal Stresses, vol. 24, no. 5, pp. 411-432, 2001.

[32] M. I. Othman, "Effect of rotation on plane waves in generalized thermo-elasticity with two relaxation times," International Journal of Solids and Structures, vol. 41, no. 11-12, pp. 2939-2956, 2004.

[33] T. He and S. Li, "A two-dimensional generalized electromagneto-thermoelastic problem for a half-space," Journal of Thermal Stresses, vol. 29, no. 7, pp. 683-698, 2006.

[34] M. I. Othman, S. Y. Atwa, and R. M. Farouk, "Generalized magneto-thermoviscoelastic plane waves under the effect of rotation without energy dissipation," International Journal of Engineering Science, vol. 46, no. 7, pp. 639-653, 2008.

[35] M. A. Ezzat and E. S. Awad, "Micropolar generalized megnatothermoelasticity with modified Ohm'sand Fourier's law," Journal of Mathematical Analysis and Applications, vol. 353, pp. 99-113, 2009.

[36] M. I. Othman, S. Y. Atwa, and R. M. Farouk, "The effect of diffusion on two-dimensional problem of generalized thermoelasticity with Green-Naghdi theory," International Communications in Heat and Mass Transfer, vol. 36, no. 8, pp. 857-864, 2009.

[37] M. I. Othman, K. Lotfy, and R. M. Farouk, "Generalized thermo-microstretch elastic medium with temperature dependent properties for different theories," Engineering Analysis with Boundary Elements, vol. 34, no. 3, pp. 229-237, 2010.

[38] M. I. Othman and K. Lotfy, "On the plane waves of generalized thermo-microstretch elastic half-space under three theories," International Communications in Heat and Mass Transfer, vol. 37, no. 2, pp. 192-200, 2010.

[39] R. Kumar, A. Miglani, and S. Kumar, "Normal mode analysis in fluid saturated porous medium," Multidiscipline Modeling in Materials and Structures, vol. 7, no. 1, pp. 29-42, 2011.

[40] R. Kumar, K. D. Sharma, and S. K. Garg, "Wave propagation in heat conducting transversely isotropic micropolar viscoelastic half space," Materials Physics and Mechanics, vol. 15, pp. 135-149, 2012.

[41] A. E. Green and P. M. Naghdi, "A re-examination of the basic postulates of thermomechanics," Proceedings of the Royal Society of London A, vol. 357, pp. 253-270, 1991.

[42] A. E. Green and P. M. Naghdi, "On undamped heat waves in an elastic solid," Journal of Thermal Stresses, vol. 15, pp. 253-264, 1992.

[43] A. E. Green and P. M. Naghdi, "Thermoelasticity without energy dissipation,” Journal of Elasticity, vol. 31, pp. 189-209, 1993. 
[44] S. Mukhopadhyay and R. Kumar, "A problem on thermoelastic interactions in an infinite medium with a cylindrical hole in generalized thermoelasticity III," Journal of Thermal Stresses, vol. 31, no. 5, pp. 455-475, 2008.

[45] M. N. Allam, K. A. Elsibai, and A. E. Abouelregal, "Electromagneto-thermoelastic problem in a thick plate using Green and Naghdi theory," International Journal of Engineering Science, vol. 47, no. 5-6, pp. 680-690, 2009.

[46] S. Chitra and M. Ciarletta, "On the harmonic vibrations in linear thermoelasticity without energy dissipation," Journal of Thermal Stresses, vol. 33, pp. 858-878, 2010.

[47] S. Chiriţă and M. Ciarletta, "Several results on uniqueness and continuous data dependence in thermoelasticity of type III," Journal of Thermal Stresses, vol. 34, no. 8, pp. 873-889, 2011.

[48] F. Passarella and V. Zampoli, "Reciprocal and variational principles in micropolar thermoelasticity of type II," Acta Mechanica, vol. 216, no. 1-4, pp. 29-36, 2011.

[49] A. C. Eringen, "Plane waves in nonlocal micropolar elasticity," International Journal of Engineering Science, vol. 22, no. 8-10, pp. 1113-1121, 1984.

[50] R. S. Dhaliwal and A. Singh, Dynamic Coupled Thermoelasticity, Hindustan Publication Corporation, New Delhi, India, 1980. 

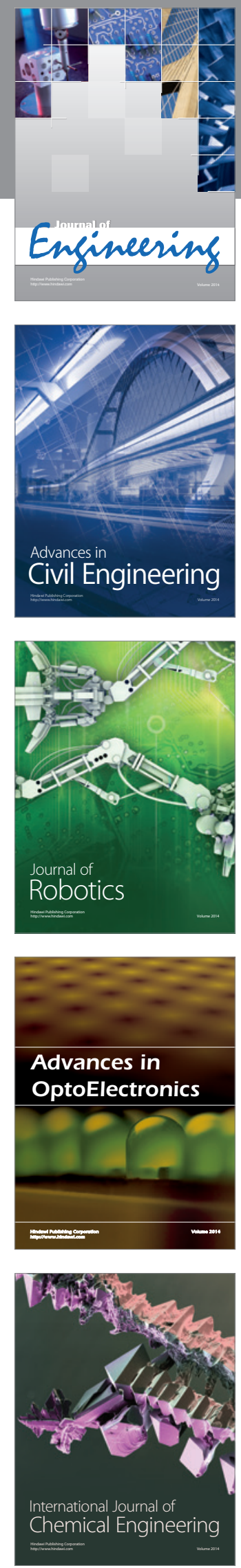

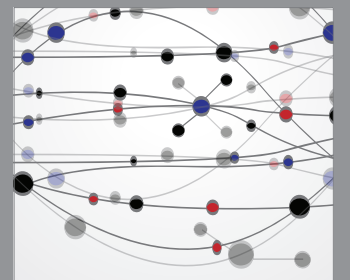

The Scientific World Journal
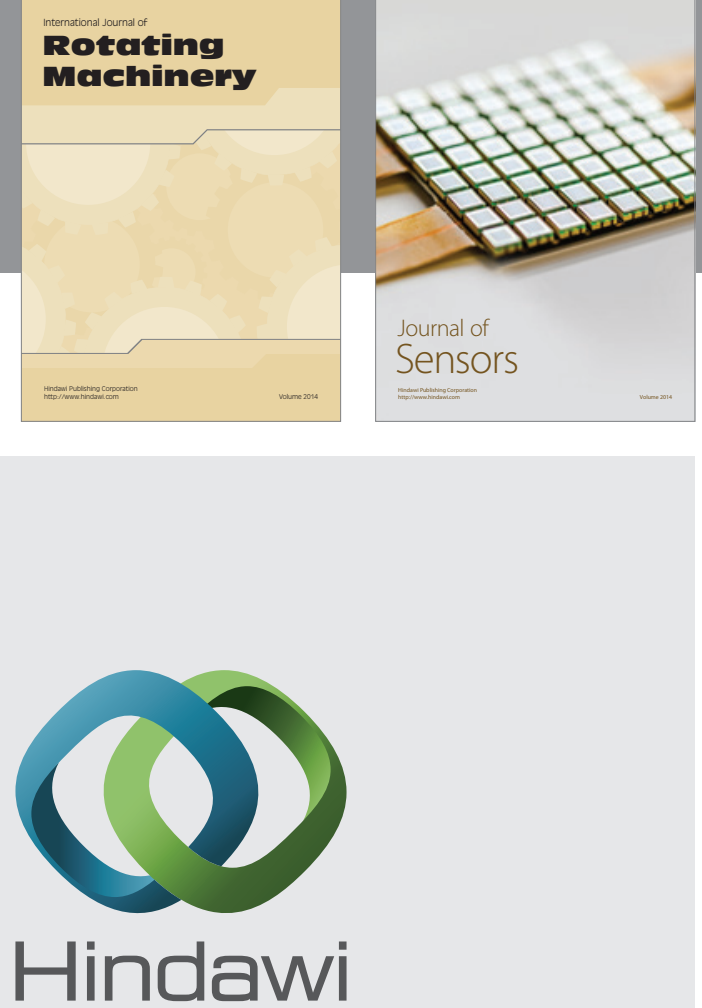

Submit your manuscripts at http://www.hindawi.com
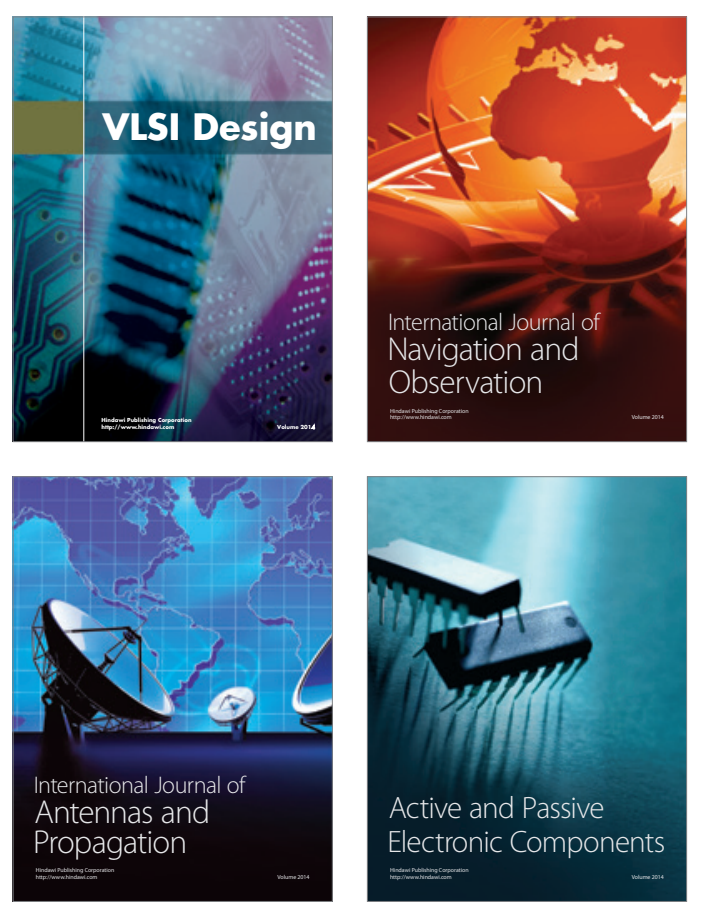
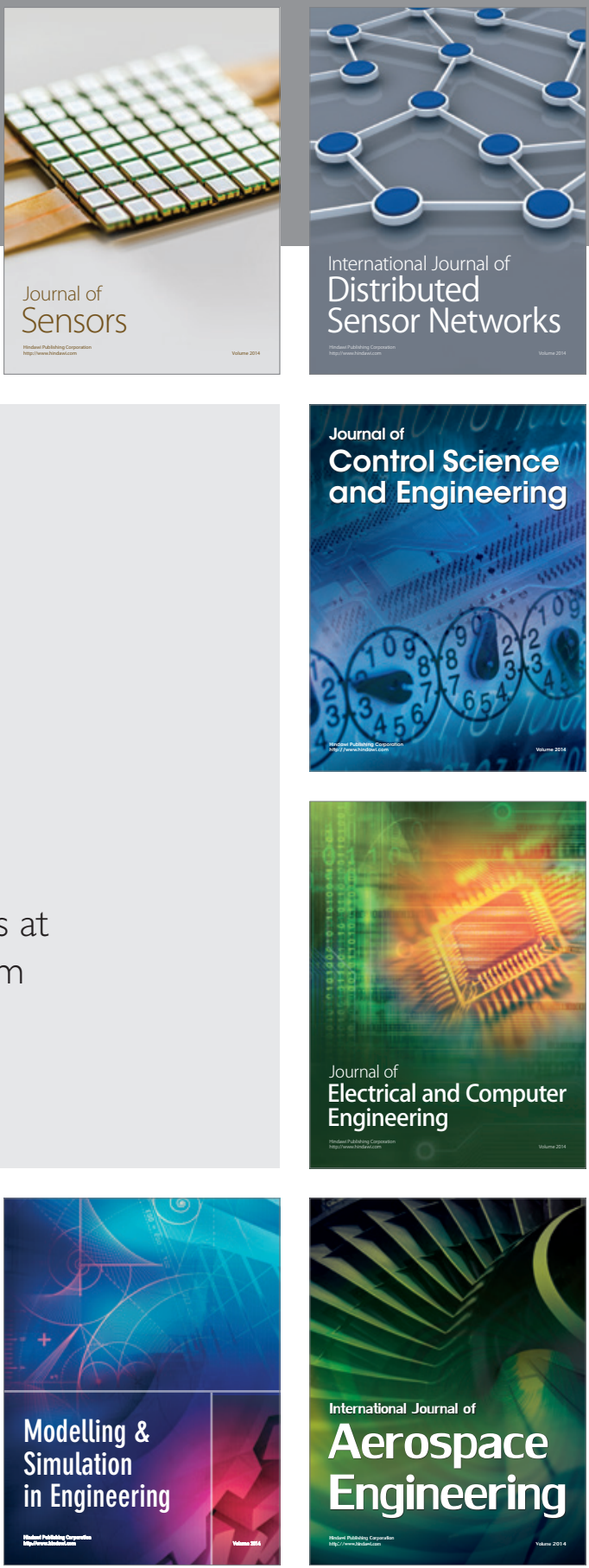

Journal of

Control Science

and Engineering
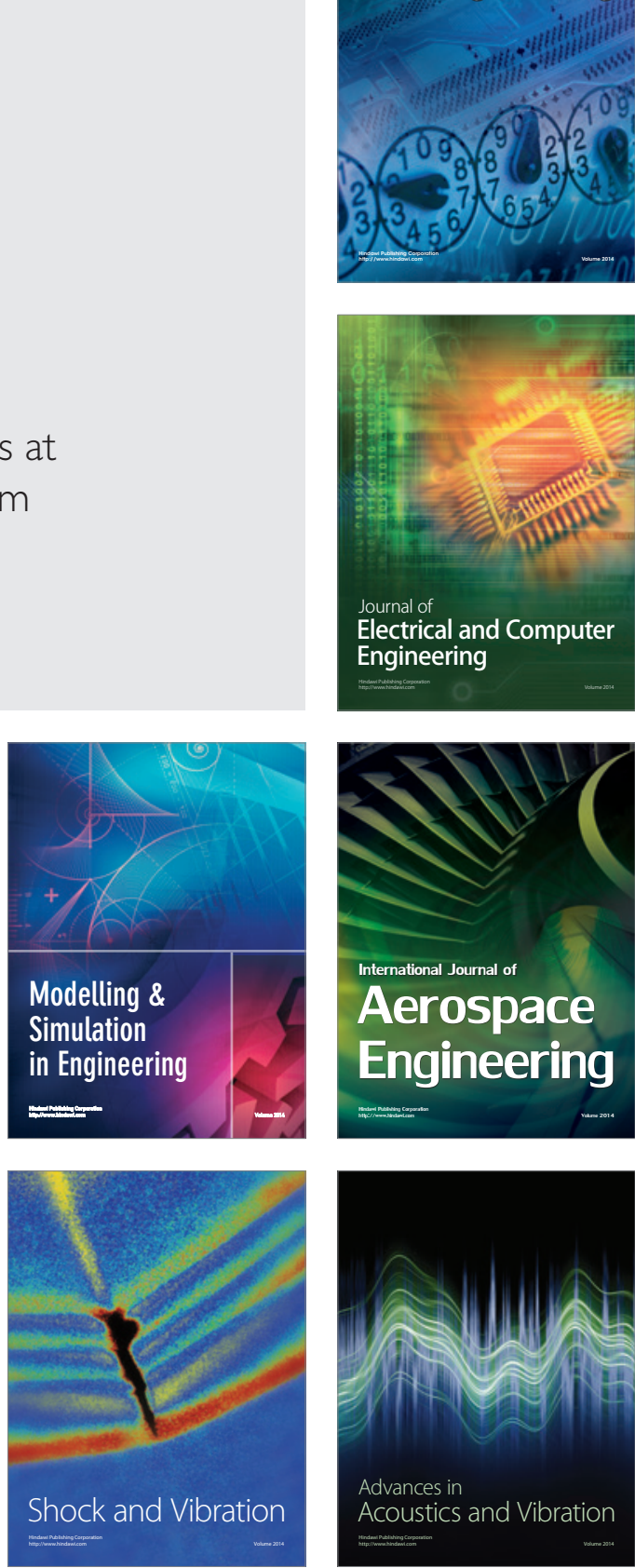